\title{
LOS PRINCIPIOS EDUCATIVOS PESTALOZZIANOS Y SU INFLUENCIA EN LA EDUCACIÓN ESPAÑOLA
}

Ma. Consolación Calderón España Ma. Isabel Corts Giner

Escribir sobre Enrique Pestalozzi (1746-1827) y su obra, y aportar algo nuevo no es tarea fácil. Nos encontramos ante el pedagogo -probablemente el único conocido en los cinco continentes- que Meylan compara con "la grandeza mítica de un Beethoven y le considera no sólo como una fuerza original de la naturaleza o mejor de la sobrenaturaleza, sino que "sin evocar sus escritos o sus hechos no se sabría escribir la historia de la civilización europea occidental ${ }^{1}$.

En estas páginas intentaremos resaltar algunos de los principios educativos sobre los que se fundamentará toda su obra y la influencia que este pensamiento ha tenido en la educación española.

Podemos afirmar plenamente que al tratar de Enrique Pestalozzi estamos frente a un humanista. Es cierto que su pensamiento, en algunos aspectos, es hijo de su época y en este sentido ciertos planteamientos suyos acerca del hombre, y especialmente de la sociedad, podríamos considerarlos superados. Sin embargo, creemos que su reflexión acerca de la esencia de la humanidad y de la educación siguen manteniendo una actualidad innegable.

El pensamiento de Pestalozzi surge en gran parte del movimiento Ilustrado; éste ponía su mirada en el Humanismo Renacentista que no hizo sino reactualizar los conceptos de la Paideia griega y de la Humanitas romana, pasados por el tamiz del cristianismo. Por ello, muchos aspectos de su humanismo, y detalles de su praxis educativa, están presentes en Platón, en Séneca, en Quintiliano, en San Agustín, en Vives, etcétera, y creemos que es importante tenerlo en cuenta, no

1 MEYLAN, L. (1956): "Heinrich Pestalozzi", en CHATEAU, J. (1956): Los grandes pedagogos, México: F.C.E., p. 203. 
para restar importancia a su pensamiento, sino para reconocer cómo, ciertos aspectos de la educación desde la antigüedad clásica, sirven de quicio para todo el pensamiento posterior.

Él hace del hombre y de la necesidad de alcanzar su perfección, su plena humanidad, el centro de su preocupación intelectual y moral, y su actividad práctica. Ante un mundo que él considera en crisis, conjura a buscar el remedio para los males que la sociedad padece allí donde se encuentra: en la restauración, en todos, de esa humanidad que es la vocación de cada hombre y de la razón de ser o el fin de la Creación: "Reconocer, mantener y procurar en cada ser la dignidad de la persona $n^{2}$. Para él, ésta será toda la educación de la humanidad. Y esta formación de la persona posee en ella misma su fuerza y su razón de ser, no al margen del mundo, sino más allá de él. Esto es lo que dice Jullien de París tras su estancia en Yverdon: «Se ocupaba de formar hombres, independientemente de los destinos que pudieran tener en el mundo" ${ }^{3}$.

Esto nos recuerda un texto de Ortega: "Los educadores, sobre todo cuando van inspirados por un afán de practicismo, piensan que lo que hay que hacer con los muchachos es prepararlos del modo más concreto posible para la vida tal cual es, dejando a un lado todas las disciplinas y modos que parecen ornamentales y superfluos. Pero la vida histórica tiene la condición de cambiar constantemente. La Historia es permanente inquietud y mutación. De modo que si se educa a un muchacho preparándolo concretamente para la vida tal cual es hoy, cuando llega a adulto se encuentra con que la vida tiene otra figura y cuanto más prácticamente preparado estuviese para lo anterior, más desajustado queda para la que tiene que vivir y en la que tiene que actuar. Es lo que he llamado el anacronismo constitutivo de la usual Pedagogía ${ }^{4}$.

${ }^{2}$ MEYLAN, L. (1956): "Heinrich Pestalozzi”, en CHATEAU, J. (1956): Los grandes pedagogos, México: F.C.E. p. 203.

${ }_{3}^{3}$ JULLIEN, M.A. (1932): Sistema de Educación de Pestalozzi. Madrid: Francisco Beltrán.

${ }^{4}$ ORTEGA Y GASSET, J. (1966): Una interpretación de la Historia Universal. Madrid: Revista de Occidente, pp. 28-29. 
Sin embargo, debemos tener en cuenta que el término $b u$ manismo puede tener diferentes significados, a veces distintos y hasta incompatibles entre sí. Los diversos conceptos de humanismos variarán según los ideales humanos puestos de relieve 5 . Por eso importa conocer esos ideales sobre los que se fundamentará toda su práctica, ya que no es la praxis la que juzga el ideal, sino el ideal a la praxis, como expresa repetidamente nuestro autor a lo largo de todas sus obras.

El sistema educativo, que de manera bastante asistemática irá elaborando Pestalozzi, tanto en sus obras como en su práctica educativa, siguiendo a $P$. Natorp ${ }^{6}$, diríamos que se estructura en torno a cinco principios básicos de su teoría educativa: naturalidad; método; intuición; desarrollo uniforme y armónico de las fuerzas humanas; y colectividad ${ }^{7}$. No podremos detenernos en cada uno, pero sí queremos señalar algunas observaciones que nos ayuden a comprenderlos mejor.

Para Pestalozzi el término "naturaleza" encierra no sólo lo instintivo, sino lo racional, moral y religioso. Es esta consideración integral del hombre, un humanismo que indica que el propio hombre es quien debe buscar dentro de sí el sentido de su humanidad; humanidad que lo transciende, que le viene dada por su experiencia como ser moral y religado a una realidad superior: Dios. Por ello Religión y Moral están íntimamente unidas en Pestalozzi y ambas se basan en la íntima experiencia de Dios en mí, a través de la propia Creación, a través de mi propio corazón.

Naturalidad para Pestalozzi no es abandonarse al instinto, a lo espontáneo, aunque esto sea el punto de partida. Natural es, en el hombre, buscar su propio perfeccionamiento y éste llega cuando "hace de lo que debe, la ley de lo que quiere" ${ }^{8}$.

${ }^{5}$ BELENGUER CALPE, E. (1987): «El humanismo en una reconceptualización radical de la educación" en Bordón, no 266, pp. 113-121.

${ }^{6}$ NATORP, P. (1931): Pestalozzi. Barcelona: Labor.

7 Cfr. JUllien, M.A. (1932): Sistema de Educación de... Ob. cit. p.152 y ss.

8 PESTALOZZI, E. (1797): Mis investigaciones sobre la marcha de la naturaleza en el género bumano. 
El punto de partida de toda instrucción humana no puede ser otro que el deseo y la íntima aspiración original de la naturaleza del hombre hacia su propio desarrollo. Es el proceso que debe seguir desde la anomía o impulso, a la autonomía o conciencia moral, pasando por la heteronomía o ley social. En este proceso de personalización, la voluntad y el esfuerzo serán esenciales, como ya lo habían advertido los clásicos, y tanto ha olvidado la Pedagogía actual.

Para Pestalozzi, la verdadera cultura humana tiene por base el desarrollo del sentido moral y de un elevado ideal de lo religioso, del amor, de la fe en la conciencia de la verdad, de la simpatía hacia lo demás... Reconoce la relación íntima existente entre cuerpo y alma, y cómo se interpretan y completan lo físico y espiritual recíprocamente?

Por ello, en 1809, cuando se dirige a sus alumnos afirma: «Lejos de nosotros la idea de convertiros en hombres que se nos parezcan, tales como la mayoría de nuestros contemporáneos. Es preciso que gracias a nuestros cuidados lleguéis a ser los hombres que vuestra naturaleza quiere que seáis, los hombres que exige lo que hay de divino y sagrado en vuestra naturaleza ${ }^{10}$.

El método para Pestalozzi es el proceso de autodesenvolvimiento del espíritu humano en avance medido y constante hasta conseguir su plenitud. Este proceso no puede ser algo basado en la técnica, sino en el desarrollo de la naturaleza y apoyado en la simple convicción "de mi amor hacia mis niños". De la relación con los niños debe surgir el método y no al revés.

El método es necesariamente algo unitario, como unitaria es la educación, por tanto no consiste en el estudio de mil diversas verdades aisladas, sino de la reconducción hacia la verdad. En esta marcha se tiene que ir de lo particular a lo general, de lo inmediato percibido y sentido a la elaboración de una serie y la reunión de estas series en un conjunto total.

\footnotetext{
${ }^{9}$ Mme. SCHRADER (1884): “Pestalozzi y Fröebel”, en B.I.L.E. nº 172, año VIII, p. 105. ${ }^{10}$ PESTAlOZZI, E. (1809): "Discurso a sus alumnos" en NATORP, P. (1931): Pestalozzi. Barcelona: Labor, p.117.
} 
Sentimiento, intelecto y gusto son los resortes fundamentales de la acción educativa ${ }^{11}$.

En este proceso de autodesarrollo hay que tener en cuenta que el niño, antes de pensar y actuar, ama y cree, que el pensamiento y la acción no se organizan sino sobre la base de una seguridad emotiva, de una cierta solidez en las relaciones afectivas. Ésta es la base del método pestalozziano, significa volver psicológica la enseñanza: basar el método en la naturaleza humana.

Al tratar de la intuición -el principio que quizás más ha trascendido del pensamiento de Pestalozzi, tanto que hay autores que le llaman el "pedagogo de la intuición" "12-, debemos advertir que para él es activa y sensible, pero que esto no significa que sea meramente externa, sino vitalidad plena de la realización. En él, la intuición no se opone a las ideas y principios generales, sino que se aproxima mucho a la función sintética del pensamiento. Es cierto que significa experiencia, pero que tiene como base una visión profunda, una observación interior del objeto mismo ${ }^{13}$.

En este mismo sentido señala a la espontaneidad como la regresión del conocimiento al "centro de todo tu ser, a ti mismo". El niño tendrá que aprender a ver, a percibir mediante la facultad de investigación que va formándose dentro de él mismo.

Al hablar del desarrollo equilibrado y armónico de las fuerzas se refiere a las espirituales, morales y físicas citadas al tratar de la naturaleza: "Cabeza, corazón y mano". Para Pestalozzi, las espirituales y morales unidas entre sí preceden a las físicas como manifestación, si bien necesitan indispensablemente de ellas.

${ }^{11}$ AbBagnano, N. y VISAlBerghi, A. (1974): Historia de la Pedagogía. México: F.C.E., p.471.

${ }^{12}$ Cfr. COMPAYRÉ, G. (1909): Pestalozzi y la educación elemental. Madrid: Librería General de Victoriano Suárez, p.58. Antes que él, Comenio y Basedow habían tratado de aplicar la intuición a la educación, pero no se popularizó hasta la aplicación de su método en las distintas escuelas que dirigió.

${ }^{13}$ NATORP, P. (1931): Pestalozzi. Ob. cit., p.83. 
Cuando Pestalozzi se plantea la instrucción del hombre, la percibe como un proceso necesariamente individual: sólo puede ser instruido por su propia voluntad. Sin embargo, como ya hemos visto, la fuerza central en el hombre es la moral y ésta señala inmediatamente a la colectividad de los seres humanos, por eso toda su Pedagogía es Social.

Para Pestalozzi, la forma primitiva y principal de la colectividad humana es la familia, por ello le da un papel tan primordial e insustituible en la educación, considerándola como el fundamento de toda pura formación natural de la humanidad. Aquí es donde el niño comienza a descubrir el mundo, los sentimientos, los valores, a Dios y a sí mismo. Aquí se colocan los cimientos de la personalidad en sus tres aspectos: intelectual, moral-religiosa y física; las organizaciones sociales deben ser de tal naturaleza que aseguren y apoyen una educación adecuada de la infancia en el hogar y en las escuelas colaborando con ellas estrechamente. En este sentido la economía, el Estado y la organización económica están en la base de la educación ${ }^{14}$.

Considera imprescindible el derecho a la educación, haciendo una fuerte crítica de la situación de la misma en su tiempo y cree que el Estado debe responsabilizarse de ella, porque es la legislación la que debe proponer la elevación de la condición moral del pueblo ${ }^{15}$.

La colectividad ciudadana es una familia de familias y Pestalozzi todavía considerará un tercer nivel social: la comunidad ideal del género humano. Los tres son, a la vez, factores y medios educativos. El orden colectivo no perjudica ni deshumaniza al hombre, sino que lo eleva a su verdadera humanidad. La educación moral en Pestalozzi se realiza con y para los demás en la realidad total de la acción colectiva de tal manera que «educar no es sino limar los eslabones de la cadena por la

\section{${ }_{14}$ Ibidem, p.121.}

${ }_{15}$ Ibidem, p.126. En este mismo sentido Natorp considera que la primera legislación en pro de una escuela nacional y pública, la prusiana, tan influida por Fichte es una consecuencia de los principios pestalozzianos. 
que todos los hombres están unidos". Él no sólo considera los valores sociales sino que intenta llevarlos a la práctica, uniendo lo que Rousseau separaba: la obediencia y la libertad, siempre que aquélla tenga sus raíces en la confianza y se base en la libertad ${ }^{16}$. Esto no es más que ese proceso en que para él radica la educación: el paso de la anomía a la autonomía.

Enrique Pestalozzi ha sido considerado padre de la Pedagogía contemporánea, reformador de la escuela popular, iniciador de la Escuela Nueva, luchador incansable por la mejora de la educación especialmente de los más desfavorecidos. Su influencia, ya durante su vida, tanto a través de sus escritos desde el "Manual de las madres" hasta el "Canto del cisne", pasando por "Leonardo y Gertrudis", "Cómo Gertrudis educa a sus hijos", etcétera, como a través de su práctica educativa en las instituciones que él dirigió personalmente, se extendió por toda Europa, permitiendo el surgimiento de escuelas a imagen y semejanza de Yverdon. Su sistema se implantó como modelo en muchas escuelas primarias y se crearon Escuelas Normales para preparar a los maestros en el espíritu pestalozziano. Educadores de Estados Unidos y países sudamericanos viajaron a Suiza para aprender su sistema y ponerlo en práctica.

No siempre, ni por todos fue comprendido: Andrés Bell, padre junto con Lancaster del sistema mutuo o lancasteriano y Pestalozzi se dieron a conocer mutuamente sus método en 1816 en Yverdon ${ }^{17}$, pero Bell no le entendió. A pesar de no estimar su procedimiento de instrucción afirmó de él que era un "hombre bueno, entusiasta y genial".

España no quedó al margen de esta influencia. Conocido es por todos su introducción por medio de dos militares suizos, Voitel y Schmeller ${ }^{8}$, pertenecientes a un destacamento

\footnotetext{
${ }^{16}$ ABBAgnANO, N. y VISAlBERGHI, A. (1974): Historia de la Pedagogía. Ob. cit., p.269.

${ }^{17}$ Cfr. COMPAYRÉ, G. (1909): Pestalozzi y la educación elemental. Madrid: Librería General de Victoriano Suárez, p.59.

${ }^{18}$ MORF, H. (1887): "Pestalozzi en España” en B.I.L.E. no 239, tomo XI, p. 54 y GIL MUäIZ, A. (1927): “Pestalozzi en España” en Revista de Pedagogía no 62 (Número extraordinario dedicado a Pestalozzi), pp.71-77.
} 
que operaba en Tarragona, cuya gran vocación pedagógica les había llevado a conocer la persona y obra de nuestro autor, y desear extenderla. Apoyados por D. José Döbely, sacerdote católico de la misma nacionalidad que los anteriores, y seguidor también de las ideas pestalozzianas. Así, en 1803, crearon una escuela en la misma Tarragona para hijos de soldados pobres.

La noticia del éxito de la escuela del regimiento tarraconense llegó a Madrid y las Reales Sociedades Económicas se interesaron por la implantación del método en sus escuelas. Todos sabemos que estas entidades tuvieron grandes iniciativas educativas y estuvieron siempre alerta y dispuestas a introducir las innovaciones pedagógicas que se producían en toda Europa ${ }^{19}$.

Especial protagonismo tuvo, en la introducción de la nueva pedagogía, la Real Sociedad Económica de Cantabria: el éxito de la escuela tarraconense animó a los representantes de la citada institución en la capital del reino a invitar a Voitel, en 1804, para que fundara una escuela en Madrid y, posteriormente, transformar en pestalozziana otra escuela que la prestigiosa Sociedad sostenía en Santander. Voitel no aceptó moverse de Tarragona hasta que el Gobierno español tomase como cosa propia y de su interés la nueva manera de enseñar. Sin embargo animó al capellán José Döbely a que marchara a Madrid y se hiciera cargo de esta propuesta.

Inmediatamente, éste fundó allí una escuela de ensayo cuyos excelentes resultados se vieron incluso antes de un año. Ante este éxito, Döbely marcha a Santander, en 1805, donde creó una Escuela Normal con 17 alumnos. Los resultados, tras el primer año de funcionamiento se pusieron de manifiesto en el examen que realizaron los normalistas en septiembreoctubre de 1806 y que fue presenciado, además de por las autoridades, por varios padres de familia deseosos de conocer

${ }^{19}$ Sobre la labor pedagógica llevada a cabo por las Reales Sociedades Económicas de Amigos del País existe una abundantísima bibliografía que aquí sería demasiado prolijo enumerar. 
los efectos del método de Pestalozzi. Los exámenes versaron sobre diferentes materias teóricas y unos ejercicios prácticos con escolares, de aplicación del sistema.

Döbely contó con el incondicional apoyo de D. Juan Andújar, secretario de la Real Sociedad Económica Cantábrica, un clérigo culto, modesto y generoso, con grandes inquietudes educativas que le llevó a traducir las obras de Pestalozzi ${ }^{20}$. Este último convencido del beneficio que para la educación española supondría la adopción del sistema de Pestalozzi en las escuelas españolas, quiso contagiar su entusiasmo al Gobierno y lo hizo por mediación de D. Francisco Amorós y Ondeano $^{21}$, secretario de Príncipe de la Paz.

Efectivamente, en agosto de 1806 se dictó un Real Decreto por el que, el 4 de noviembre del mismo año, se inauguró en Madrid una escuela según los principios de Pestalozzi, a expensas del Estado, formalizándose además un Reglamento ${ }^{22}$. La escuela estaba destinada en principio a hijos de oficiales, después se amplió, pudiendo admitir hasta 100 alumnos. Uno de los objetivos principales que perseguía era funcionar como Escuela Normal para que los maestros ahí formados, extendieran el sistema por todo el país.

Voitel fue nombrado Director y Schmeller, Ayudante Primero. La inauguración del Real Instituto Pestalozziano Militar se realizó con gran solemnidad el día citado y el discurso de apertura lo pronunció su director. Los alumnos contaban desde 5 a 14 años y el curriculum comprendía Cálculo, Medida y Número, Historia y Geografía, Español, Francés e Inglés, Aritmética y Geometría, Gimnasia, Dibujo y Religión (de la que se encargó D. Juan de Andújar). Pronto necesitaron un nuevo

\footnotetext{
${ }^{20}$ MORF, H. (1887): “Pestalozzi en España” en B.I.L.E. no 239, tomo XI, p. 54 y GIL MUÑIZ, A. (1927): "Pestalozzi en España" en Revista de Pedagogía no 62 (Número extraordinario dedicado a Pestalozzi), pp.71-77.

${ }^{21}$ GIL MUÑIZ, A. (1927): "Pestalozzi en España” en Revista de Pedagogía, no 62 , pp.71-78.

${ }^{22}$ La legislación relacionada con el tema aparece en LUZURIAGA, L. (1916): Documentos para la Historia escolar de España. Madrid: Junta para la ampliación de estudios e investigaciones científicas. Volumen II, pp.27-81.
} 
ayudante y fue propuesto Studer ${ }^{23}$, que tras formarse en Yverdon aceptó el nombramiento.

La comunicación entre estos educadores y el mismo Pestalozzi fue directa en esta primera época y, como dice un informe enviado a Godoy en 1807, el método Pestalozziano «hace en nuestra patria progresos por extremos rápidos y felices" tanto, que se educa según él al infante D. Francisco de Paula.

El Príncipe de la Paz mandó difundir una Circular a todas las Reales Sociedades Económicas para que enviasen al nuevo centro a maestros, eclesiásticos y pedagogos a fin de que aprendieran el sistema, habiendo reservado para ello 50 plazas. La respuesta fue tan masiva que pronto no hubo cabida para todos los que acudieron a la llamada.

Sin embargo, a pesar de el primer éxito, estas realizaciones no fueron duraderas: los azares políticos, envidias y soberbia personal de algunos de sus protagonistas ${ }^{24}$, no dejaron que las instituciones madrileña y santanderina siguieran adelante. En este sentido fue ilustrativa la crítica que Josef de Narganes, Catedrático de Ideología y de Literatura Española en el colegio de Soreze, hizo de esta Institución: tras elogiar el sistema pestalozziano como el más filosófico, el más conforme a la naturaleza y el más propio para formar la razón, creía que su implantación en España, concretamente en este establecimiento, no había sido acertada ya que, en la realidad, los que allí se educaban pertenecían a la alta sociedad y sugería "que si el Gobierno piensa de veras en adoptar el método de Pestalozzi y hacerlo general a toda la nación, forme un hospicio, no de hijos de señores, sino de pobres abandonados e hijos de la sociedad, y ensaye en ellos este sistema de educación y cuando vea que el fruto corresponde a las esperanzas, tendrá un método de educación primaria seguro y se hallará con un número considerable de maestros que podrán ir a establecerlo

${ }^{23}$ MORF, H. (1887): "Pestalozzi en España”, en B.I.L.E. nำ 243, año XI, p.89.

${ }^{24}$ Ibidem, pp.120-121. 
en los pueblos de las provincias, hasta que llegue a las más pequeñas aldeas ${ }^{25}$.

Así, repentinamente, el 18 de enero de 1808, a los 14 meses de su creación fue suprimido el Real Instituto Pestalozziano por una Real Orden del 3 de enero de 1808. No obstante, a lo largo de todo el siglo y del siguiente podemos decir que la presencia de Pestalozzi ha estado en todos los movimientos a favor de la mejora de la educación en España.

Además de las escuelas de Santander y Madrid, tenemos noticias de la creación en otros lugares de España de escuelas que seguían el método pestalozziano, bajo el amparo de las Reales Sociedades Económicas. Podemos citar como ejemplos a Soria ${ }^{26}$, Palencia (escuela de párvulos) ${ }^{27}$ o Valencia, donde la Comisión de primera Educación de la Real Sociedad Económica de la capital levantina, con fecha de 1807, recalcó la necesidad de suministrar lo necesario para que los jóvenes recibieran la primera instrucción sin salir de su lugar de origen y una de las soluciones aportadas era la de fomentar los métodos pestalozzianos ${ }^{28}$.

En Sevilla, en esta primera época no hemos encontrado datos sobre el funcionamiento de ninguna escuela que siguiera el citado sistema, pero sí tenemos noticia de haber enviado a algunos maestros al Real Instituto Pestalozziano de

25 NARGANES, J. de (1809): Tres cartas sobre los vicios de la Instrucción Pública en España y proyecto de un Plan para su reforma. Madrid, p.95 y ss. ${ }^{26}$ PÉREZ-RIOJA, J.A. (1972): "La Sociedad Económica Numantina de los Amigos del País de Soria y su provincia" en Patronato "José María Cuadrado". Pleno. (1972. San Sebastián): Las Reales Sociedades Económicas de Amigos del País y su obra. Comunicaciones presentadas en el pleno de la Asamblea, celebrada en San Sebastián durante los días 9 al 11 de diciembre de 1971. San Sebastián. Patronato "José María Cuadrado". Consejo Superior de Investigaciones Científicas, pp.339-346.

${ }^{27}$ SÁNCHEZ GARCÍA, J.L. (1991): La Sociedad Económica de Amigos del País de Palencia (Ss. XVIII-XX). Palencia: Excma. Diputación Provincial, p.127.

${ }^{28}$ Archivo de la Real Sociedad Económica de Amigos del País de Valencia. C47, III. Ed. no. 4. Citado por LÓPEZ TORRIJOS, M. y MAYORDOMO, A. (1984): "Labor educativa de la Real Sociedad Económica de Amigos del País de Valencia (Aproximación histórica)" en Revista de Ciencias de la Educación, no. 118 , p. 185 . 
Madrid a aprenderlo ${ }^{29}$; en su rica biblioteca se conservan varios manuales en los que se ofrece una explicación del método ${ }^{30}$.

Del mismo modo hemos hallado otros manuales en los que se refleja la difusión del método pestalozziano en España durante el siglo XIX ${ }^{31}$.

Pablo Montesino fue fiel seguidor de Pestalozzi. En 1840, a la vuelta del exilio, publicó el "Manual para los maestros de escuelas infantiles" donde recogió las enseñanzas del de Yverdon. Su nombramiento como Director General de Instrucción Pública fue decisivo para la propagación de sus ideas tanto en las escuelas que se crearon, siguiendo el sistema pestalozziano, en Guadalajara y Alcoy, como en la Escuela Normal Central de Madrid que dirigiría él mismo ${ }^{32}$.

Esta escuela abrió sus puertas el 29 de enero de 1939. Recibió a 30 alumnos aunque después aumentaron hasta 50 provenientes de todas las provincias españolas. Montesino supo inspirar un gran entusiasmo a profesores y alumnos. Los nuevos aires educativos que él había vivido en el extranjero, entre ellos el del espíritu pestalozziano, estuvieron presentes en su organización y sobre todo en su espíritu, dándole un papel primordial a la formación moral y religiosa sin abandonar la pedagógica. Estos primeros alumnos de la Escuela Normal se

${ }^{29}$ Archivo de la Real Sociedad Económica Sevillana de Amigos del País. Acta del 7 de marzo de 1807.

${ }^{30}$ Entre los textos aparecidos en nuestro país en aquellos momentos podemos citar el denominado: Noticia de las providencias tomadas por el Gobierno para observar el nuevo método de la enseñanza primaria de Enrique Pestalozzi, editado en Madrid en la Imprenta Real el año 1807. En el citado libro, que comienza explicando el nuevo método de Pestalozzi y su aplicación a las enseñanzas de la lectura, la Lengua, la Geografía y la Historia Natural, se recoge el Reglamento de la Escuela Militar Pestalozziana de Madrid. Contiene también el informe de la Comisión nombrada para observar los resultados del método, número de alumnos, distribución, número de maestros, ejercicios de los alumnos, faltas de asistencia de los mismos y progresos observados en la enseñanza por la aplicación del método.

${ }^{31}$ Cfr. CHAVANNES, A., (1807): Exposición del método elemental de Henrique Pestalozzi. Madrid: Imprenta de Gómez Fuentenebro, traducida al castellano por Don Eugenio de Luque.

32 TURIN, Y. (1967): La educación y la escuela en España de 1874 a 1902. Madrid: Aguilar, p.176. 
consideraban destinados a llevar por toda España los beneficios de la civilización, y esta idea los animaba al trabajo y les hacía conseguir rapidísimos progresos. Y es que cualquier cambio en la educación española tenía que pasar necesariamente por una mejor preparación de sus maestros. Esta experiencia de la Escuela Normal Central dio lugar a que se crearan las primeras Escuelas Normales provinciales donde ejercieron sus enseñanzas los que habían sido alumnos de la Central ${ }^{33}$.

En Sevilla hubo otros dos focos de influencia de Pestalozzi a finales del siglo XIX y principios del XX. El primero de ellos fue tratado en un trabajo por la Doctora doña Ana María Montero ${ }^{34}$, bajo el título de "Influencias de la pedagogía pestalozziana en la enseñanza sevillana”. El segundo se trató de un trabajo titulado "Un proyecto de reforma de la enseñanza pública, que costea el Municipio. Sevilla 1900", elaborado por una de las autoras de esta comunicación y publicado en las actas del II Coloquio de Historia de la Educación celebrado en 1983 en Valencia, con el título de "Escolarización y sociedad en la España contemporánea (1808-1970),35.

La influencia de Pestalozzi en Montesino aparece clara, además, en su «Manual para los maestros de párvulos" (1840). En él preconiza los procedimientos intuitivos, juegos, variedad en los ejercicios, método activo, lecciones de cosas e insiste, siguiendo a Pestalozzi, en que la educación de los niños pequeños debe estar en manos de la mujer, al mismo tiempo que reconoce que, para ello, es preciso transformar la educación femenina, que él cree más necesaria que la de los hombres para lograr el bienestar social.

33 Cfr. GIL DE ZÁRATE, A. (1855): De la Instrucción Pública en España. Madrid: Imprenta del Colegio de Sordomudos, Vol. I, p.262 y ss.

34 Profesora del Departamento de Teoría e Historia de la Educación y Pedagogía Social de la Universidad de Sevilla.

${ }^{35}$ Cfr. CORTS GINER, Ma. I. (1983): "Proyecto de reforma de la enseñanza pública que costea el Ayuntamiento de Sevilla, presentada por el Concejal D. Carlos Cañal" en Escolarización y sociedad en la España contemporánea (1808-1970). Valencia: Universidad. 
La intervención de Pestalozzi en las ideas de los hombres de la I.L.E., a través de Krause y Fröebel fue grande. Es de todos conocido que muchas de las ideas del pensamiento de Giner de los Ríos se fundamentaban en él, aunque luego se establecieran ciertas disparidades. A pesar de ellas creemos que se puede afirmar que Giner "está en la línea de los continuadores de Pestalozzi” y que en sus orientaciones pedagógicas para la Institución Libre de Enseñanza utilizará muchas de las innovaciones del sistema pestalozziano ${ }^{36}$.

La admiración de Rafael María de Labra y de Joaquín Costa hacia Pestalozzi también es patente. Para el primero, Pestalozzi junto con Fröebel es el apóstol, promotor y fundador de la escuela primaria contemporánea, quien puso en práctica la obra de Comenio y de Rousseau, admirable la de éste último pero "que no ha podido perder el carácter de una obra incompleta discutida constantemente en la esfera de la teoría, ${ }^{37}$.

Costa, en su intervención en el Congreso Pedagógico de 1882, que tenía como tema a debatir la intuición, uno de los pilares del sistema de Pestalozzi, declara que la aplicación del método intuitivo "viene a transformar el concepto que hasta aquí se tenía de la escuela y a levantar la bandera de una escuela nueva". Para él, las lecciones de cosas, las excursiones instructivas, consideradas hasta entonces como procedimientos auxiliares, deben ser lo principal de todo. Por otra parte, la dimensión social de la educación, tan clara en Pestalozzi, es también uno de los principios fundamentales en Costa: "La escuela si quiere cumplir la noble misión que tiene confiada en nuestro siglo, no puede quedar aislada del contexto en el que vive, no puede considerarse en un invernadero, tiene que actuar al aire libre, aspirar la vida a raudales difundiéndose como la sangre por los conductos y arterias del cuerpo social, ${ }^{38}$.

${ }^{36}$ GÓMEZ GARCÍA, Ma. N. (1983): Educación y Pedagogía en el pensamiento de Giner de los Ríos. Sevilla: Universidad, pp.91-97.

${ }^{37}$ LABRA, R. Mำ (1887): “Pestalozzi y Fröebel” en B.I.L.E., no 246, año XI, p.131. ${ }^{38}$ COSTA, J.: "Actas del Congreso Pedagógico de 1882" en BATANAZ PALOMARES, L. (1982): La educación española en la crisis de fin de siglo. Córdoba: Diputación Provincial, p.201. 
Si tomamos los Congresos Pedagógicos que tuvieron lugar en España a finales del siglo XIX y principios del XX como foros representativos de la inquietud por la mejora regeneración y modernización de la educación, también aquí vemos presente el espíritu de Pestalozzi.

Convocados siguiendo el ejemplo de Alemania, pionera en este movimiento, y de Francia y Bélgica, y promovidos en primer lugar por la Sociedad del Fomento de las Artes a la que se fueron añadiendo, inicialmente y de forma aislada maestros, personalidades, preocupadas por la educación y después entidades de muy diverso tipo, como las Reales Sociedades Económicas, la I.L.E., el obrero español, la liga de contribuyentes, etcétera, los temas pestalozzianos estuvieron presentes en todos ellos. Así ya en el primero, el de 1882, el tercer tema propuesto fue el de la intuición en las escuelas primarias ${ }^{39}$.

También el Congreso de Pontevedra de 1887 tuvo como uno de los temas centrales el de la intuición, su fundamento, su empleo y si debía limitarse a la enseñanza primaria. Cuando se trata de la escuela de párvulos, son los métodos de Montesino y Fröebel los que se ofrecen como modelo y tras ellos, lógicamente, el de Pestalozzi en el que se han fundamentado los dos anteriores.

Al hablar de la escuela primaria en el Congreso de 1882, Carderera afirma: "Montesino como Pestalozzi sabían que el secreto de la primera educación está en sostener y dirigir indirectamente la actividad, tendencia irresistible del niño. Ejercicio de los sentidos corporales, de la inteligencia, de la voluntad, del sentimiento moral y religioso en armonía con las disposiciones y necesidades de los niños, en eso consiste la educación ${ }^{40}$.

Pero el camino de las reformas educativas debe pasar forzosamente por quienes la llevan a cabo, por los maestros como medios indispensables para una renovación de su formación que influyera positivamente en la escuela.

${ }^{39}$ Ibidem, p.48.

${ }^{40}$ Ibidem, p.148. 
Ya hemos hablado de cómo Montesino, en la Escuela Normal Central, intentó poner en práctica muchos aspectos del sistema pestalozziano. Sin embargo, este ejemplo no prendió como era de desear en las Escuelas Normales que se fueron creando, y aunque fueron sin duda un medio de mejora de la formación de los maestros, no consiguieron los frutos deseados. Por ello en los Congresos, especialmente en el de 1892, al tratar el tema de la formación de los maestros, volvió a surgir el modelo pestalozziano, el maestro como auxiliar necesario, como animador, nunca como protagonista, la necesidad del estudio de la Pedagogía y de la Psicología, así como de prácticas, conferencias pedagógicas, excursiones científicas, Museos Pedagógicos, etcétera ${ }^{41}$.

Si repasamos la legislación española sobre la instrucción primaria en los siglos XIX y XX, aunque sin entrar a fondo, ya que ello requeriría un profundo estudio -aunque las distintas leyes provengan de ideologías diferentes, al plantear innovaciones educativas-, podemos percibir en ella los ecos del espíritu de la nueva educación propugnada por Pestalozzi.

Ya cuando Quintana, en su Informe, afirma que la instrucción pública es «el arte de poner a los hombres en todo su valor tanto para ellos como para sus semejantes", vemos claramente la línea del pensamiento de nuestro autor, al igual que cuando aconseja seguir a la naturaleza. Desde un punto de vista más práctico, la introducción del Dibujo, junto a la Aritmética y Geometría, en el curriculum, es también una influencia pestalozziana ${ }^{42}$.

En 1821 se realizó el proyecto de un Reglamento General de Instrucción Primaria ${ }^{43}$, el primero en España, y que no llegó a ponerse en práctica por avatares políticos. En él se recogían las ideas de Quintana, pero quería ir más allá. Esto se ve

\footnotetext{
${ }^{41}$ Ibidem, p.141.

${ }^{42}$ QUINTANA, J. "Informe de la Junta creada por la regencia para proponer los medios de proveer al arreglo de los diversos ramos de la Instrucción Pública" en Historia de la Educación en España, (1979) Tomo 1‥ Madrid: M.E.C., pp.373-414. ${ }^{43}$ Cfr. Proyecto de Reglamento General de Primera Enseñanza que se ha de observan en todas las Escuelas de Primeras Letras de la monarquía española. (1922). Madrid: Imprenta de M. Repulles.
} 
claramente en el Plan metódico para su aplicación, publicado por una Real Orden de 30 de septiembre de 1822, en el que se realizó un análisis del método de Pestalozzi observado por Vallejo, uno de los autores del citado Plan, en el propio Instituto Pestalozziano ${ }^{44}$.

También en el Plan General de Instrucción Pública de 1836, estuvo clara la influencia de Pestalozzi a través de Montesino y todavía más en el Reglamento de las Escuelas Públicas de Instrucción Primaria elemental del 26 de noviembre de 1836, llamado también Reglamento Montesino, de fuerte carácter innovador: amplió las materias de enseñanza introduciendo firmemente el Dibujo, recomendó el uso del sistema simultáneo, señalaba la conveniencia de una graduación de los niños y de la enseñanza como propugnaba Pestalozzi, incluso hablaba de una graduación flexible. Abogó por la lectura comprensiva frente a la mecánica desde el principio, y del aprendizaje simultáneo de ésta y de la escritura, restaba importancia al libro de texto y subrayaba la labor del maestro, concedía una gran importancia a la formación religiosa y moral y aconsejaba que fueran las mujeres quienes se ocuparan de la formación de los párvulos ${ }^{45}$.

En la Ley Moyano, primera Ley General de Educación española, que planteaba la educación obligatoria de todos los niños, se siguieron en parte algunas de las innovaciones presentadas en el Reglamento anterior de Montesino: mantenía la importancia de la enseñanza moral: "formar el corazón de los niños", consagraba la existencia en el curriculum del Dibujo, tanto para niños como para niñas, añadiendo por primera vez el Canto y dejaba relativa libertad a los maestros en la elección de los textos.

En el Real Decreto del 8 de junio de 1910, en el que se establecía la graduación de las enseñanzas en todas las

${ }^{44}$ Este Plan metódico está recogido en el texto «Exposición sobre el estado de la enseñanza pública hecho a las Cortes”. (1822). Madrid: Albán y Cía.

${ }^{45}$ LÓPEZ DEL CASTILLO, Ma. T. (1982): "Planes y programas escolares de la legislación española", en Bordón, nºs. 242-243, pp.139 у 154. 
escuelas, resuenan los ecos de Pestalozzi. De nuevo volvemos a apreciar su influencia en la Circular del 12 de enero de 1932 dada por el Gobierno de la II República ${ }^{46}$. En ella se abogó por una escuela activa aboliendo la libresca, potenciando los trabajos manuales, presentando la necesidad de inculcar el espíritu colectivo, ya que el trabajo individual sería tanto más útil como mejor sirviera a la comunidad; trataba de establecer un estrecho contacto entre escuela y realidad a través de paseos, excursiones, lecturas, etcétera, y recomendaba construir una ceñida relación con los padres mediante reuniones o las mismas misiones pedagógicas. Sin embargo, en algunos aspectos de su espíritu, se apartó del pensamiento de Pestalozzi, por ejemplo en el tema del laicismo.

El Plan de estudios del 28 de octubre de 1937, que se impuso en la zona republicana, siguió las líneas del anterior. El que continuó en la llamada zona nacional, impuesto por una Circular de 5 de marzo de 1938, estableció como eje de la primera enseñanza la educación religiosa, la educación patriótica, la educación cívica y la educación física. Se abogó por una enseñanza globalizada y se crearon diferentes tipos de escuela según el medio: rurales, urbanas, marítimas, ambulantes, etcétera.

En los distintos planes de instrucción Primaria del período franquista, se intentaron recoger en algunos aspectos, y al menos en la letra de la Ley, las líneas de la educación nueva. Así por ejemplo, en los cuestionarios publicados el 1o. de diciembre de 1953, los primeros programas de nuestra legislación a pesar de sus posibles defectos. Sin embargo Adolfo Maillo hizo referencia a la enseñanza concreta viva y activa, a la negación de todo verbalismo, de todo memorismo, partir del círculo de ideas del niño, recurrir a las realidades vitales como el mejor material de enseñanza, utilizar la intuición y la acción como medios didácticos, plantear la lección como un conjunto de actividades de acción entre maestros y niños ${ }^{47}$.

${ }^{46}$ GACETA DE MADRID, 14 de enero de 1932.

${ }^{47}$ LÓPEZ DEL CASTILLO, Mำ T. (1982): “Planes y programas...”. Ob. cit., pp.185-188. 
También los cuestionarios nacionales de enseñanza primaria elaborados bajo la dirección de Juan Manuel Moreno en 1965, establecían aspectos novedosos en cuanto a actividad, necesidad de creación de hábitos, destrezas y actitudes, etcétera, en la línea de las ideas de Pestalozzi.

En 1970 la segunda Ley General de Educación produjo un cambio en el sistema educativo español casi revolucionario: la educación personalizada, la importancia de la expresión plástica y la expresión dinámica, los métodos activos, la conexión entre áreas de expresión y áreas de experiencia, el trabajo en equipo, la agrupación flexible de alumnos, la programación por objetivos, el estímulo a la iniciativa y creatividad marcarían un nuevo hito en la educación española. Todas estas características pueden observarse en el sistema pestalozziano ${ }^{48}$.

La Ley del 70 planteaba como objetivo la formación humana integral, el desarrollo armónico de la personalidad y la preparación para el ejercicio responsable de la libertad inspirados en el concepto cristiano de la vida y en la tradición y cultura patria (art. 1), la extensión de esta educación a todos, el hacer un hecho la gratuidad de la enseñanza y que contempla la necesidad de desarrollar programas de educación familiar para proporcionar a los padres y tutores orientaciones relacionadas con su misión educadora y de cooperación con los centros docentes, ideas que son básicas en la doctrina del pedagogo suizo ${ }^{49}$.

Todo este recorrido (que somos conscientes apenas aporta nada nuevo a lo ya conocido por todos), sería deseable que sirviera para tomar conciencia de que en España ha habido una influencia, unas veces pujante, otras más solapada, de este gran maestro, cumpliéndose lo que en el prólogo de su obra "El manual de las madres", escribía en palabras premonitorias: "Las formas de mi método perecerán, pero el espíritu que las anima, el espíritu de mi método, sobrevivirá”.

\footnotetext{
${ }^{48}$ Cfr. entre otras las siguientes obras: CHAVANNES, A. (1807): Exposición del método elemental de Henrique Pestalozzi. Madrid: Imprenta de Gómez Fuentenebro; JUlliEN, M.A. (1932): Sistema de Educación de Pestalozzi. Madrid: Francisco Beltrán; COMPAYRÉ, G. (1909): Pestalozzi y la educación elemental. Madrid: Librería General de Victoriano Suárez, etcétera.

${ }^{49}$ Ibidem.
} 
En efecto, es en los momentos de crisis -cuando se plantea la educación como medio de salvar al hombre, de regenerarlo-, cuando se vuelve la mirada hacia Pestalozzi. Sin embargo, creemos que en esta influencia ha pesado muchas veces más la praxis que la filosofía que le ilumina y que han sobrevivido más las formas del método que su espíritu.

La Ley General de Educación de 1970, sirvió de base a la L.O.G.S.E. (1990), tal como lo reconocen los elaboradores de la nueva norma legal ${ }^{50}$, aunque haya entre ambas leyes claras y profundas diferencias motivadas por la ideología subyacente y por las nuevas exigencias educativas de la sociedad española.

En la Ley de Ordenación General del Sistema Educativo (L.O.G.S.E.), si tenemos en cuenta sus planteamientos de tipo didáctico y metodológico, creemos observar algunos ecos de los principios pestalozzianos -teniendo en cuenta los cambios operados en la sociedad desde principios del siglo XIX, época en la que empezó a extenderse el sistema del citado pedagogo, y nuestros días, ya en las puertas del tercer milenio-, a pesar de que en la enumeración de los principios de intervención educativa, elaborados en el Diseño Curricular Base de la citada Ley, se afirma que los principios psicopedagógicos que subyacen en el mismo "no pueden identificarse con ninguna teoría en concreto, más bien con enfoques presentes en los distintos marcos teóricos ${ }^{51}$.

Fijándonos en las etapas correspondientes a la educación infantil y la primaria, períodos de los que se ocupó fundamentalmente el pedagogo helvético, queremos enfatizar una primera diferencia: el cambio producido en la realidad familiar actual que ha llevado a nuestros legisladores contemporáneos, entre otras razones, a la conveniencia de implantar un nivel educativo que abarca de 0 a 6 años, por lo que el papel que Pestalozzi atribuía a los padres y fundamentalmente a la

${ }^{50}$ MINISTERIO DE EDUCACIÓN Y CIENCIA (1989): Libro Blanco para la reforma del Sistema Educativo. Madrid: M.E.C., p.13.

${ }^{51}$ MINISTERIO DE EDUCACIÓN Y CIENCIA (1989): Diseño Curricular Base. Educación Infantil. Madrid: M.E.C., p.31. 
madre queda, en muchos casos, transferido a los/as maestros/as de esta etapa, aunque de alguna manera se considera el papel de la familia y su necesaria colaboración con el centro educativo: "Los centros docentes de educación infantil cooperarán estrechamente con los padres o tutores a fin de tener en cuenta la responsabilidad de éstos en dicha etapa educativa" (art. 7). "Las familias y el centro docente comparten los objetivos de acompañar, guiar y estimular el desarrollo psicológico del niño a través de diferentes experiencias educativas que favorecen que este desarrollo se realice de manera integral, ${ }^{52}$. Igual ocurre con la etapa de Educación Primaria: "La educación primaria debe desarrollarse en estrecho contacto con las familias de los alumnos ${ }^{53}$.

El primer eco que creemos descubrir de influencia pestalozziana, es la intención de los legisladores españoles es su declaración de que "la mejora del sistema educativo supone ofrecer un marco curricular... sin rupturas entre las distintas etapas ${ }^{34}$, lo que enlaza con la idea de Pestalozzi de que la educación debía ser continua y sin intervalos ${ }^{55}$.

El artículo 6 de la L.O.G.S.E. señala expresamente que "la educación infantil (...) contribuirá al desarrollo físico, intelectual, afectivo, social y moral de los niños ${ }^{56}$. Ésta es la idea pestalozziana de que en la educación deben intervenir "la cabeza, el corazón y las manos", es decir, las capacidades intelectuales, morales y físicas.

En el Diseño Curricular Base, el primer principio de la intervención educativa se refiere a la necesidad de partir del nivel de desarrollo del niño. Esto se identifica con el principio

${ }^{52}$ MINISTERIO DE EDUCACIÓN Y CIENCIA. (1989): Libro Blanco para la Reforma del Sistema educativo. Madrid: M.E.C., p.103.

${ }^{53}$ Ibidem, p.115.

${ }^{54}$ MINISTERIO DE EDUCACIÓN Y CIENCIA. (1989): Diseño Curricular Base. Educación Infantil. Madrid: M.E.C., p.19.

${ }^{55}$ Cfr. JUllien, Ob. cit. p.152 y ss.

${ }^{56}$ Estas intenciones aparecen desarrolladas en el Diseño Curricular Base. Cfr. MINISTERIO DE EDUCACIÓN Y CIENCIA. (1989): Diseño Curricular Base. Educación Infantil. Madrid: M.E.C., pp.71-72. 
establecido ya por Rousseau, y seguido por Pestalozzi, de que la educación debe basarse en el desarrollo de la naturaleza, en el conocimiento propio que será el centro del que deba partir la esencia de la instrucción humana, y Pestalozzi resalta como punto de partida el desarrollo de la potencia pensante y cognoscitiva y no el objeto a conocer.

Como segundo principio, aparece la necesidad de asegurar "la construcción de aprendizajes significativos (...) con lo que se asegura la funcionalidad de lo aprendido, ${ }^{57}$. El tercer principio consiste en que "realicen aprendizajes significativos por sí solos, es decir, que sean capaces de aprender a aprender ${ }^{58}$ y ese aprendizaje significativo supone "modificar los esquemas de conocimiento que el alumno posee" ( $4^{\underline{o}}$ principio) ${ }^{59}$. Los esquemas los irá modificando, no por acumulación de conocimientos, sino concatenando lo nuevo con lo ya sabido. Estas modificaciones se llevan a efecto por medio de "una intensa actividad por parte del alumno" ( $5^{\circ}$ principio $)^{60}$, ideas que, como ya hemos indicado antes y todos conocemos, están expresadas en la doctrina de Pestalozzi: la instrucción para Pestalozzi se basa en el hacer ${ }^{61}$, en la facultad de investigación que va formándose y desarrollándose dentro del niño. En él y en la L.O.G.S.E. están presentes los principios didácticos de la actividad, del rechazo del verbalismo y la necesidad de una enseñanza práctica en todos los campos.

Pestalozzi expresa la necesidad de lo que él llama "educación particular", es decir, la estrecha interacción profesoralumno que llevará a una educación individualizada ${ }^{62}$. En

${ }^{57}$ MINISTERIO DE EDUCACIÓN Y CIENCIA. (1989): Diseño Curricular Base. Ob. cit., pp.32-33 y 91.

${ }^{58}$ Ibidem, p.33.

59 Idem.

${ }^{60}$ Ibidem, p.34.

${ }^{61}$ Todo el sistema pestalozziano se basa en el hacer: el niño debe construir, por ejemplo, sus propios mapas, al principio sencillos y más tarde irá añadiendo diversos elementos: cadenas de montañas, ríos, etcétera. Cfr. JULLIEN, M.A. (1932): Sistema de Educación de... Ob. cit. p.152 y ss.

${ }^{62}$ Cfr. JULliEN, M.A. (1932): Sistema de Educación de... Ob. cit., p.154 y ss. Expone las ideas de la graduación en todo el proceso de educación e instrucción. 
este sentido enfoca la L.O.G.S.E. la función tutorial como una actividad orientadora vinculada "estrecha e indisolublemente con la práctica docente, ${ }^{63}$. La intervención es considerada en dicha Ley como un "proceso de interactividad profesor-alumno o alumno-alumno", contemplándose las relaciones de tipo tutorial también como el proceso en el que el alumno cumple la función de profesor con otro compañero ${ }^{64}$, lo que ha demostrado ser de gran ayuda para el desarrollo de la enseñanza-aprendizaje. Pestalozzi también pensaba que este tipo de relación alumno-alumno, podía ser mucho más útil en la formación del carácter que la que pudieran recibir de sus propios profesores ${ }^{65}$.

Entre los primeros objetivos nombrados en el "Manual de las madres", está el de que los niños logren el conocimiento de su propio cuerpo, para lo cual propone diversos ejerci$\operatorname{cios}^{66}$, lo que aparece recogido igualmente en la L.O.G.S.E. (art. 8) ${ }^{67}$. Ejemplos de ejercicios prácticos para conseguir este conocimiento aparecen en "Ministerio de Educación y Ciencia (1989): "Ejemplificaciones del Diseño Curricular Base. Educación Infantil y Primaria”". Madrid: M.E.C., y especificados en los bloques de contenido que se desarrollan en el Diseño Curricular Base ${ }^{68}$.

Lengua y Matemáticas son los dos ejes fundamentales sobre los que la L.O.G.S.E. establece la instrucción básica. Recordemos que también lo eran para Pestalozzi. Para él, número,

\footnotetext{
${ }^{63}$ Ibidem, pp.225-227.

${ }^{64}$ MINISTERIO DE EDUCACIÓN Y CIENCIA. (1989): Diseño Curricular Base... Ob. cit, p.35.

${ }^{65}$ Cfr. JULliEN, M.A. (1932): Sistema de Educación de... Ob. cit., p.152 y ss.

${ }^{66}$ Cfr. CHAVANNES, A. (1807): Exposición del método elemental de Henrique Pestalozzi, Ob. cit, pp.18-24.

${ }^{67}$ L.O.G.S.E. Art. 8: La educación infantil contribuirá a desarrollar en los niños las siguientes capacidades: a) Conocer su propio cuerpo y sus posibilidades de acción. b) Relacionarse con los demás a través de las distintas formas de expresión y comunicación...

Cfr. MINISTERIO DE EDUCACIÓN Y CIENCIA (1989): Diseño curricular Base... Ob. cit, pp.139-140.

${ }^{68}$ Cfr. MINISTERIO DE EDUCACIÓN Y CIENCIA (1989): Diseño curricular Base... Ob. cit, pp.125-131.
} 
forma y palabra eran los puntos de partida del conocimiento.

El dominio de la Lengua está muy relacionado en Pestalozzi con el conocimiento de su entorno ya que en el nombrado «Manual de la madres" se presenta como método de aprendizaje: una vez conocidos los sonidos de las sílabas, deberá aprender a nombrar las distintas partes de su cuerpo ${ }^{69}$, es decir, nombrando lo más cercano a él mismo. En la L.O.G.S.E. se especifica que una de las capacidades a desarrollar en la educación infantil es la de "relacionarse con los demás a través de las distintas formas de expresión gestual y corporal, del lenguaje verbal, la expresión plástica, el lenguaje escrito.... ${ }^{70}$. No se clarifica la forma por la que el alumno aprenderá el lenguaje verbal, pero en los ejemplos ofrecidos para llevar a cabo la teoría, se incluye el conocimiento, por parte del niño, de su propio cuerpo, evaluando positivamente la expresión de distintos sonidos ${ }^{71}$.

Pestalozzi pensaba que la educación debía ser gradual y progresiva, es decir, analítica. En su Instituto, cada uno de los cursos se componía de grados, en los que se les iban enseñando los conocimientos con mayor profundidad, a medida que se ensanchaba el círculo y se ampliaban sus capacidades intelectuales $^{72}$. Como sabemos, la educación primaria está estructurada, en la L.O.G.S.E., en tres ciclos: 6-8 años, 8-10 años y 10-12 años (artículo 12), recogiendo, de forma gradual y progresiva, los aprendizajes de distintas materias ${ }^{73}$.La Religión (obligada para el centro y voluntaria para los alumnos) aparece en: "Ministerio de Educación y Ciencia (1989): "Libro Blanco..."” Ob. cit., p. 112. Los planteamientos son globalizadores e interdisciplinares, tal como Pestalozzi preconizaba.

${ }^{69}$ Cfr. CHAVANNES, A. (1807): Exposición del método elemental de Henrique Pestalozzi. Ob. cit., p.26-34.

${ }^{70}$ Cfr. MINISTERIO DE EDUCACIÓN Y CIENCIA (1989): Diseño curricular Base... Ob. cit, p.164.

${ }^{71}$ MINISTERIO DE EDUCACIÓN Y CIENCIA (1989): Libro Blanco... Ob. cit., pp.104-105.

${ }^{72}$ Cfr. JUllien, M.A. (1932): Sistema de Educación de... Ob. cit., p.157 y ss.

${ }_{33}$ Conocimiento del medio natural social y cultural, Educación artística (Musical y plástica), Educación física, Lengua castellana y la lengua oficial de la correspondiente comunidad autónoma y Literatura, Lenguas extranjeras, Matemáticas. 
En el diseño sobre la Educación Infantil, indica la Ley española vigente que: "Se basará en las experiencias, las actividades y el juego..." (art. 9, apartado 5) y en "los descubrimientos junto a otros compañeros.... ${ }^{74}$. Respecto del juego y su papel educador, queremos destacar cómo esta idea, muy presente en Pestalozzi, sin embargo y como otras muchas, viene repitiéndose desde los clásicos. Ya Platón afirma, en «La República", que es en el juego donde el niño muestra su espontaneidad, donde expresa abiertamente su personalidad. También, entre otros, nuestro gran humanista Vives se manifestará en este mismo sentido.

Pestalozzi señalaba la conveniencia de usar pocos libros en la enseñanza primaria, considerando que la instrucción dada por los objetos mismos es superior a la que pueda aprender el educando en los libros durante sus primeros años de vida. Para él, la enseñanza de las cosas debe preceder a la de las palabras. Ver, oír y hacer, deben ser anteriores a juzgar e inducir y también el principio de todo saber y de toda educación no es el arte ni los libros, sino la vida. En la escuela secundaria sí debía utilizarlos para obtener una parte de su cultura de las obras de autores que legaron a la humanidad sus trabajos e investigaciones ${ }^{75}$.

En el "Libro Blanco", que expone el desarrollo de la L.O.G.S.E., no se rechaza el libro de texto, pero se propone que el predominio de éste no sea exclusivo, ya que ello «hace inviable un planteamiento de globalización e interdisciplinariedad ${ }^{76}$, debiendo facilitar la administración la utilización de otros materiales como fuente de información y aprendizaje (videos, periódicos, revistas, etcétera $)^{77}$. Es obvio señalar que estos medios no existían a principios del siglo XIX pero en el sistema pestalozziano se impulsaba, como ya hemos indicado en líneas

\footnotetext{
${ }^{74}$ MINISTERIO DE EDUCACIÓN Y CIENCIA (1989): Libro Blanco... Ob. cit., p.107.

${ }^{75}$ Cfr. JULLIEN, M.A. (1932): Sistema de Educación de... Ob. cit., p.157.

${ }^{76}$ Cfr. MINISTERIO DE EDUCACIÓN Y CIENCIA (1989): Libro Blanco... Ob. cit., p.113.

${ }^{77}$ Idem.
} 
anteriores, que el niño debe construir sus elementos de trabajo, lo que le sirve también como fuente de información.

Pestalozzi estaba convencido de que la naturaleza es la gran maestra de la vida. Aspiraba a que los niños estudiaran los minerales, plantas, animales, etcétera, en la misma realidad, no en los libros ni en figuras o dibujos en ellos presentadas. Con este sistema el alumno se acostumbra a observar los objetos en sí mismos y a observar y expresar con exactitud y precisión ${ }^{78}$, idea que está reflejada en la L.O.G.S.E.: “La educación primaria contribuirá a desarrollar en los niños las siguientes capacidades: (...) Conocer las características fundamentales de su medio físico, social y cultural y las posibilidades de acción en el mismo" 7 .

Con la potenciación del trabajo junto a sus compañeros, la Ley está intentando abrir a los alumnos el espíritu de trabajo y de cooperación. Pestalozzi se planteaba la escuela como una potencia social educadora, que no sustituyera a la familia, pero que pudiera aportar valores distintos y complementarios.

Uno de los aspectos más enriquecedores de la L.O.G.S.E., es el tratamiento que hace de la educación física y artística para la educación primaria, tan abandonadas en la educación española, viéndolas como aspectos fundamentales para una educación integral tal y como también Pestalozzi y, antes de él otros autores clásicos, habían propugnado, y que quedan desarrolladas ampliamente en el Diseño Curricular.

Podríamos seguir insistiendo - un trabajo más amplio nos obligaría a hacerlo-, sobre los aciertos de esta Ley en los cuales directa o indirectamente creemos ver una influencia pestalozziana. Sin embargo y, quizás como reflexión final de nuestra comunicación, queremos resaltar, en respuesta al interrogante que planteábamos al principio, algunas importantes discordancias que encontramos entre la teoría básica educativa de Pestalozzi y los principios que sustentan nuestra Ley.

${ }^{78}$ Cfr. JUllien, M.A. (1932): Sistema de Educación de... Ob. cit. p.152 y ss.

${ }^{79}$ Art. 13, apartado g. 
Creemos que la L.O.G.S.E. es una Ley hecha demasiado al hilo de los tiempos cosa que, en principio, no tendría por qué ser negativa, pero que lo es en cuanto que, a nuestro modo de ver, intenta configurar la personalidad humana de acuerdo con una sociedad muy determinada, más que de acuerdo a unos valores humanos que a veces pueden ir en contra de lo establecido. Como respaldo de esta afirmación nuestra, vemos que en el "Libro Blanco", al plantear los objetivos fundamentales de la educación, se sitúa en primer lugar la socialización de los educandos, una socialización identificada con la preparación para una vida en democracia y, en segundo lugar, favorecer el desarrollo integral de los alumnos ${ }^{80}$, aunque luego advierta que ambos objetivos son complementarios.

Es en este sentido en el que vemos una falta de planteamiento serio de la formación humana. Es cierto que en la L.O.G.S.E. se habla de una formación personalizada y de una formación integral. Sin embargo estos términos no son unívocos, como advertíamos al principio de nuestra comunicación. También Pestalozzi nos habla de una educación personalizada e integral, pero creemos que en un sentido bastante diferente de la L.O.G.S.E.

Para Pestalozzi, toda educación tiene que partir de un conocimiento profundo, reflexivo e interiorizado de nuestro propio ser y de lo que nos rodea, y esto no lo vemos claro en una Ley que ha marginado aquellas materias, como la Filosofía, la Historia, las Lenguas Clásicas, que son las que hacen, precisamente, pensar y reflexionar al hombre sobre su propio yo, su origen, su ser, el punto de partida desde el cual tiene que proyectarse y sobre el fundamento, devenir y futuro de la sociedad en la que vive.

Junto a esto, echamos de menos una mayor consideración de la educación moral, piedra angular del sistema pestalozziano y, según él, «envoltura de todas las demás materias

${ }^{80}$ Cfr. MINISTERIO DE EDUCACIÓN Y CIENCIA. (1989): Libro Blanco... Ob. cit., p.94. 
educativas». Es cierto que en la letra de la L.O.G.S.E. está presente la formación moral, que en ella tiene un tratamiento como materia transversal y, por supuesto, así ha de ser, aunque entrañe para su práctica tantos problemas -entre ellos, y como fundamental, el de la propia formación moral de los maestros, de cuya preparación casi han desaparecido, no sólo la Ética, sino las materias básicas de formación personal como la Antropología, la Filosofía de la Educación, la Historia de la Educación, etcétera-; sin embargo, carece de un espacio específico con unos contenidos morales claros y precisos que el niño tiene que experimentar, conocer y hacer suyos, como ya se ha planteado en las reformas acometidas en países como Suecia y Gran Bretaña.

Echamos de menos una Pedagogía de la voluntad y del esfuerzo, de "hacer de lo que debo, la ley de lo que quiero", como propugnaba Pestalozzi, sin lo cual la educación moral no es posible.

En España, comienza a hacerse patente un clamor generalizado en este sentido por parte tanto de intelectuales, como de educadores y de las propias familias ${ }^{81}$. La sociedad actual, a pesar de sus logros, está muy lejos de ser una sociedad que favorezca el desarrollo integral del hombre. La falta de valores morales, tanto en la vida pública como privada, la violencia creciente en las relaciones sociales, manifestada alarmantemente desde edades muy tempranas, el relativismo, la trivialización del pensamiento, la crisis de la institución familiar, etcétera, todo ello exige volver la mirada hacia la esencia del ser humano, de la persona, de lo que verdaderamente le hará posible alcanzar su plenitud y su felicidad profunda.

\footnotetext{
${ }^{81}$ No enumeramos, porque resultaría exhaustivo, los artículos que a lo largo del curso pasado y en el presente, han sido publicados en la prensa diaria y en revistas especializadas en las que se solicita una revisión de objetivos y valores, sobre todo.
} 
Por tanto es tarea de todos cambiar nuestro mundo y la educación tiene en ello un papel primordial, como se está reconociendo desde todos los sectores.

Es necesario hacer una revisión, no tanto de la metodología, como de los principios básicos sobre los que se debe sostener la educación. Y en este sentido creemos que hoy, a los hombres y mujeres de principios del siglo XXI, Pestalozzi tiene muchas cosas qué decirnos. 